An International Journal of Optimization and Control: Theories \& Applications ISSN:2146-0957 eISSN:2146-5703

\title{
A comparison of some control strategies for a non-integer order tuberculosis model
}

\author{
Tuğba Akman Yıldız (D) \\ Department of Logistics Management, University of Turkish Aeronautical Association, Ankara, Turkey \\ tr.tugba.akman@gmail.com
}

\section{ARTICLE INFO}

Article History:

Received 30 July 2018

Accepted 19 February 2019

Available 15 April 2019

Keywords:

Tuberculosis

Optimal control

Fractional derivative

AMS Classification 2010:

49K99; 34A08; 37N25

\author{
ABSTRACT
}

The aim of this paper is to investigate some optimal control strategies for a generalized tuberculosis model consisting of four compartments. We construct the model with the use of Caputo time fractional derivative. Contribution of distancing control, latent case finding control, case holding control and their combinations are discussed and the optimality system is obtained based on the Hamiltonian principle. Additionally, we prove that the solution is non-negative and bounded from above. We present some illustrative examples to determine the most effective strategy to minimize the number of infected people and maximize the number of susceptible individuals. Moreover, we discuss the contribution of the Caputo derivative and the order of the fractional derivative to efficiency of the control strategies.

(cc) BY

\section{Introduction}

Tuberculosis (TB) is a threatening bacterial disease caused by Mycobacterium tuberculosis. It is the ninth leading cause of death in the world and about 1.3 million people died due to TB in 2016 according to 2017 TB report of World Health Organization (WHO) [1]. Dynamics of TB is slowly varying when compared to other epidemiological diseases. Additionally, infected people do not show any symptoms of the disease for years and approximately $5-10 \%$ of the latently infected people become an active TB sufferer [2, while $90-95 \%$ of people remain latent and they do not infect other people at this stage. For latent individuals to be an active TB sufferer depends on endogenous reactivation or exogenous reinfection [3]. WHO and the United Nations aim to end TB throughout the world, so they set the target as "a $90 \%$ reduction in TB deaths and an $80 \%$ reduction in TB incidence (new cases per year) by 2030, compared with 2015" [1].

Before setting some goals to end such a disease, the use of mathematical models to understand the dynamics of the disease has gained a special interest for a while [4]. In the literature, there are different models and optimal intervention strategies that are formulated based on different aspects of the disease. Dynamics of TB was firstly formulated by Waller and his colleagues in 1962 through a system of difference equations [5]. Since then, time evolution of the disease has been investigated. To model the disease, the population has been divided into some groups/classes, for example, representing susceptible, infectious, latent, vaccinated and recovered individuals [6]. A very basic model consisting of susceptible, infectious and recovered individuals has been proposed in [7]. As a different discussion, one-strain and two-strain TB models have been constructed to examine the antibiotic-resistant $\mathrm{TB}$ case as a result of incomplete treatment [8]. A long latent period of TB has been formulated with a distributed delay in [9. A model incorporating seasonal changes has been constructed with the use of periodic coefficients in [10].

Fractional differentiation and integration operators, which are the generalization of classical 
integer-order counterparts, capture memory effects due to their nonlocal nature [11]. Recently, it has been observed that the fractional order models with fixed-order may not be capable of expressing some real world phenomena and the need for variable order fractional operators are used [12 14]. It is a useful tool to develop suitable models for describing real-world problems which cannot be expressed by using integer-order differential equations. For example, a model for rubella disease has been formulated with the use of non-local and non-singular fractional derivatives in [15]. A fractional TB model with time delay representing the required time to commencement of treatment and diagnosis has been studied in [16], while uniform asymptotic stability of a TB model with Caputo derivative has been investigated in [17]. These studies mainly concern a single disease. Models for the interaction of two diseases can be mentioned, too. Co-infection of HIV and TB has been discussed in [18, while impact of diabetes to TB has been investigated in [19].

Optimal control problems (OCP) can be used to find an intervention or treatment strategy for realworld problems. For example, new therapy protocols can be found with the use of optimal control strategies for cancerous tumor growth model in [20]. The optimal intervention strategy among vaccination and treatment can be decided by minimizing the transmission of malaria disease [21]. Moreover, spread of Ebola disease can be controlled with vaccination of the susceptible population [22]. On the other hand, the infection level of HIV and the overall treatment cost is minimized and the duration of therapy is optimized in [23]. A fractional optimal control problem (FOCP) has been proposed for two-strain TB model in [24]. Optimal control theory is used to reduce the cost of interventions in case of reinfection and post-exposure interventions, and the sensitivity of the reproduction number has been investigated in 25]. The optimal intervention strategy has been set to minimize the number of infected individuals with the control of exogenous reinfection through the use of chemoprophylaxis 26. As a case study, the cost of TB treatment in Cameroon has been set as a cost functional and the control functions represent education-diagnosis campaign and chemoprophylaxis treatment [27]. In addition, a two-strain TB model has been taken as a constraint and the latent and infectious groups with the resistant-strain TB have been minimized with two types of treatments [28. For a review on optimal control of TB models, we refer the reader to the study [29].
In this study, we consider the recent paper of Kim and his colleagues [30] where optimal intervention strategies to reduce the number of infected people in Philippines have been compared and some values for TB incidence for 2035 have been predicted. TB model in that study has been constructed for susceptible, high-risk latent, low-risk latent and infectious individuals using integer-order derivative. We propose a generalized TB model with the use of Caputo time fractional derivative since symptoms of TB may not be observed quickly. On the contrary, latent period of the disease might last for years. In the paper 30, the aim is to minimize the number of high-risk latent and infected people with the cost of applying the controls. In addition to these, we maximize the number of susceptible people and the control strategies are fixed as distancing control, latent case finding control, case holding control and their combination. We record the values of susceptible $S(t)$ and infected $I(t)$ individuals for different fractional orders in 2035 and we observe the contribution of the order. In addition, we calculate the reduction and increase in $I$ and $S$, respectively. At the end, we comment on the choice of the optimal intervention strategy for Philippines by underlying the contribution of the fractional derivative.

The rest of the paper is organized as follows: In Section 2, we mention some properties and definitions for Caputo fractional derivative. In Section [3, we describe the generalized TB model, show that the solution is non-negative and bounded from above and propose the FOCP together with the optimality system. In Section 4 , we present some numerical results to compare different strategies. Then, the paper ends with summary and conclusion.

\section{Preliminaries}

Fractional differentiation and integration operators, which are the generalization of classical integer-order counterparts, are capable of capturing memory effects due to their nonlocal nature. In the literature, several fractional derivatives have been defined. One of the mostly used fractional differentiation operators is Caputo derivative.

We define the (left) Caputo fractional differentiation operator for $0<q<1$ as 11

$$
{ }_{a}^{C} \mathcal{D}_{t}^{q} g(t)=\frac{1}{\Gamma(1-q)} \int_{a}^{t} \frac{g^{\prime}(s)}{(t-s)^{q}} d s .
$$

The corresponding right differentiation operator is given by 


$$
{ }_{t}^{C} \mathcal{D}_{b}^{q} g(t)=-\frac{1}{\Gamma(1-q)} \int_{t}^{b} \frac{g^{\prime}(s)}{(s-t)^{q}} d s
$$

To prove that the solution of the model is nonnegative, we need the following lemma and corollary related to generalized mean value theorem [31:

Lemma 1. Let $g(x) \in C[a, b]$ and ${ }_{a}^{C} \mathcal{D}_{t}^{q} g(t) \in$ $C(a, b]$ for $0<q \leq 1$. Then, for $a \leq s \leq b$ and $\forall x \in(a, b]$, the following estimate holds:

$$
g(x)=g(a)+\frac{1}{\Gamma(q)}\left({ }_{a}^{C} \mathcal{D}_{t}^{q} g\right)(s)(x-a)^{q} .
$$

Corollary 1. Let $g(x) \in C[a, b]$ and ${ }_{a}^{C} \mathcal{D}_{t}^{q} g(t) \in$ $C(a, b]$ for $0<q \leq 1$. If ${ }_{a}^{C} \mathcal{D}_{t}^{q} g(t)$ is non-negative $\forall x \in(a, b)$, then $g(x)$ is non-decreasing for each $x \in[a, b]$. If ${ }_{a}^{C} \mathcal{D}_{t}^{q} g(t)$ is non-positive $\forall x \in(a, b)$, then $g(x)$ is non-increasing for each $x \in[a, b]$.

To show that the solution is bounded from above, we need the Laplace transform. The Laplace transform of the (left) Caputo derivative is obtained as

$$
\mathcal{L}\left\{{ }_{a}^{C} \mathcal{D}_{t}^{q} g(t)\right\}=s^{q} G(s)-g(0) s^{q-1} .
$$

Moreover, the Laplace transform of the MittagLeffler function is given by

$$
\mathcal{L}\left\{t^{p-1} E_{q, p}\left(-a t^{q}\right)\right\}=\frac{s^{q-p}}{s^{q}+a},
$$

where $E_{q, p}(z)=\sum_{i=0}^{\infty} \frac{z^{i}}{\Gamma(q i+p)}$.

\section{Fractional optimal control problem}

In this study, we generalize a tuberculosis model given in the study [30] with the use of Caputo time fractional derivative and investigate the contribution of Caputo derivative in terms of a FOCP. The model is composed of four epidemiological classes: susceptible, $S(t)$; high-risk latent, $H(t)$; infectious or active TB, $I(t)$ and low-risk latent, $L(t)$. Indeed, the total population size is represented by $N(t)=S(t)+H(t)+I(t)+L(t)$.

The OCP given in the study [30] offers a way to minimize the population of infectious and highrisk latent classes. In this current study, a FOCP is constructed to minimize the population of infectious and high-risk latent classes while maximizing the number of susceptible people together with the cost of implementing three different control strategies as

$$
\begin{aligned}
& \min _{\left(u_{1}, u_{2}, u_{3}\right) \in U_{a d}} J\left(u_{1}, u_{2}, u_{3}\right) \\
= & \int_{0}^{t_{f}}\left(H(t)+I(t)-S(t)+\frac{\omega_{1}}{2} u_{1}^{2}(t)\right. \\
& \left.+\frac{\omega_{2}}{2} u_{2}^{2}(t)+\frac{\omega_{3}}{2} u_{3}^{2}(t)\right) d t
\end{aligned}
$$

subject to

$$
\left\{\begin{aligned}
{ }_{0}^{C} \mathcal{D}_{t}^{q} S(t) & =b^{q} N(t)-\beta^{q}\left(1-u_{1}(t)\right) \frac{S(t) I(t)}{N(t)} \\
& -\mu^{q} S(t), \\
{ }_{0}^{C} \mathcal{D}_{t}^{q} H(t) & =\beta^{q}\left(1-u_{1}(t)\right) \frac{S(t) I(t)}{N(t)} \\
& -\left(\alpha^{q}\left(1+u_{2}(t)\right)+\kappa^{q}+\mu^{q}\right) H(t) \\
& +p r^{q}\left(1-u_{3}(t)\right) I(t), \\
{ }_{0}^{C} \mathcal{D}_{t}^{q} I(t) & =\kappa^{q} H(t)-\left(r^{q}+\mu^{q}+d^{q}\right) I(t), \\
{ }_{0}^{C} \mathcal{D}_{t}^{q} L(t) & =\left(1-p\left(1-u_{3}(t)\right)\right) r^{q} I(t) \\
& +\alpha^{q}\left(1+u_{2}(t)\right) H(t)-\mu^{q} L(t),
\end{aligned}\right.
$$

with $S(0)=S_{0}, H(0)=H_{0}, I(0)=I_{0}, L(0)=L_{0}$ where the admissible space of controls is given by 30 .

$$
\begin{aligned}
& U_{a d}=\left\{\left(u_{1}(t), u_{2}(t), u_{3}(t)\right) \mid u_{1}(t), u_{2}(t), u_{3}(t)\right. \\
& \text { are measurable with } \\
& \left.0.05 \leq u_{1}(t), u_{2}(t), u_{3}(t) \leq 0.95, t \in\left[0, t_{f}\right]\right\} .
\end{aligned}
$$

In other words, the optimal control $\left(u_{1}^{*}, u_{2}^{*}, u_{3}^{*}\right) \in$ $U_{a d}$ is required so that

$J\left(u_{1}^{*}, u_{2}^{*}, u_{3}^{*}\right)=\min _{\left(u_{1}, u_{2}, u_{3}\right) \in U_{a d}} J\left(u_{1}, u_{2}, u_{3}\right)$ is reached.

Remark 1. By adding the equations in the model (7) side by side, the dynamical model for the total population is obtained as

${ }_{0}^{C} \mathcal{D}_{t}^{q} N(t)=\left(b^{q}-\mu^{q}\right) N(t)-d^{q} I(t), \quad N(0)=N_{0}$.

In Table 1, we mention the values/units of the parameters in the model 30].

Table 1. Parameters in the model.

\begin{tabular}{lll}
\hline Parameter & Description (Units) & Value \\
\hline \hline$b$ & Effective birth rate $\left(\mathrm{yr}^{-1}\right)$ & 0.0442 \\
\hline$\mu$ & Natural death rate $\left(\mathrm{yr}^{-1}\right)$ & 0.0235 \\
\hline$\beta$ & Transmission rate $\left(\mathrm{yr}^{-1}\right)$ & 11.7345 \\
\hline$\alpha$ & Progression rate from $H$ to $L\left(\mathrm{yr}^{-1}\right)$ & 0.2077 \\
\hline$\kappa$ & Progression rate from $H$ to $I\left(\mathrm{yr}^{-1}\right)$ & 0.0294 \\
\hline$r$ & Treatment rate $\left(\mathrm{yr}^{-1}\right)$ & 0.2906 \\
\hline$d$ & TB-induced mortality rate $\left(\mathrm{yr}^{-1}\right)$ & 0.05 \\
\hline$p$ & Treatment failure probability & 0.2 \\
\hline
\end{tabular}


Remark 2. We follow the study [32] to take $q^{\text {th }}$ powers of the parameters, which have temporal units, to eliminate dimension mismatch.

\subsection{Non-negative and bounded solution}

In this section, we will prove that the solution to (7) is non-negative and bounded from above. To do this, we fix the controls as $u_{1}(t)=u_{1}$, $u_{2}(t)=u_{2}$ and $u_{3}(t)=u_{3}$.

Theorem 1. Let $(S(t), H(t), I(t), L(t))$ be the solution to the model (17). Then, the solution remains in $\mathbb{R}_{+}^{4}$.

Proof. We observe that the the model leads to the following inequalities:

$$
\begin{aligned}
\left.{ }_{0}^{C} \mathcal{D}_{t}^{q} S(t)\right|_{S=0} & =b^{q} N \geq 0, \\
\left.{ }_{0}^{C} \mathcal{D}_{t}^{q} H(t)\right|_{H=0} & =\left(1-u_{1}(t)\right) \beta^{q} \frac{S I}{N} \\
& +p r^{q}\left(1-u_{3}(t)\right) I \geq 0, \\
\left.{ }_{0}^{C} \mathcal{D}_{t}^{q} I(t)\right|_{I=0} & =\kappa^{q} H(t) \geq 0, \\
\left.{ }_{0}^{C} \mathcal{D}_{t}^{q} L(t)\right|_{L=0} & =\left(1-\left(1-u_{3}(t)\right) p\right) r^{q} I \\
& +\left(1+u_{2}(t)\right) \alpha^{q} H(t) \geq 0 .
\end{aligned}
$$

By Corollary 1, the solution remains in $\mathbb{R}_{+}^{4}$.

Theorem 2. Let $(S(t), H(t), I(t), L(t))$ be the solution to the model (7). The solution is bounded from above.

Proof. Firstly, we add the equations in (7) to reach (8). Then, we observe that the inequality

$$
{ }_{0}^{C} \mathcal{D}_{t}^{q} N(t) \leq\left(b^{q}-\mu^{q}\right) N(t),
$$

holds. Then, we take the Laplace transform of both sides in (10) to get the relation

$$
\lambda^{q} \mathcal{L}\{N(t)\}-\lambda^{q-1} N(0) \leq\left(b^{q}-\mu^{q}\right) \mathcal{L}\{N(t)\} .
$$

Arranging (11), we reach the inequality

$$
\mathcal{L}\{N(t)\} \leq \frac{\lambda^{q-1}}{\lambda^{q}-b^{q}+\mu^{q}} N(0) .
$$

Using (15) and taking inverse Laplace of both side, we obtain the relation

$$
N(t) \leq E_{q, 1}\left(-\left(\mu^{q}-b^{q}\right) t^{q}\right) N(0) \leq C N(0),
$$

since $E_{q, 1}\left(-\left(\mu^{q}-b^{q}\right) t^{q}\right) \leq C$ for some real number $C$. Then, we derive that the total population is bounded from above which leads the solution of (77) to be bounded from above.

\subsection{Optimality system}

We proceed with the characterization of the FOCP (647). To obtain the optimality system associated to the optimal control $\mathcal{U}^{*}=\left(u_{1}^{*}, u_{2}^{*}, u_{3}^{*}\right)$, we use Pontryagin's maximum principle [33]. We construct the Hamiltonian as

$$
\begin{aligned}
\mathcal{H}(\mathcal{X}, \mathcal{U}, \mathcal{P}) & =\left(H(t)+I(t)-S(t)+\frac{\omega_{1}}{2} u_{1}^{2}(t)\right. \\
& \left.+\frac{\omega_{2}}{2} u_{2}^{2}(t)+\frac{\omega_{3}}{2} u_{3}^{2}(t)\right) \\
& +\lambda_{1}^{T}(t)\left({ }_{0}^{C} \mathcal{D}_{t}^{q} S(t)\right)+\lambda_{2}^{T}(t)\left({ }_{0}^{C} \mathcal{D}_{t}^{q} H(t)\right) \\
& +\lambda_{3}^{T}(t)\left({ }_{0}^{C} \mathcal{D}_{t}^{q} I(t)\right)+\lambda_{4}^{T}(t)\left({ }_{0}^{C} \mathcal{D}_{t}^{q} L(t)\right) \\
& +\lambda_{5}^{T}(t)\left({ }_{0}^{C} \mathcal{D}_{t}^{q} N(t)\right),
\end{aligned}
$$

where $\lambda_{i}(t)^{\prime}$ s are the co-state (adjoint) variables for $1 \leq i \leq 5$. Then, the state equation (7) is obtained by the equation

$$
\left.\frac{\partial \mathcal{H}}{\partial \mathcal{P}}\right|_{\mathcal{U}^{*}}={ }_{0} \mathcal{D}_{t}^{q} \mathcal{X}(t)
$$

where the adjoint equation is derived as

$$
\left.\frac{\partial \mathcal{H}}{\partial \mathcal{X}}\right|_{\mathcal{U}^{*}}={ }_{t} \mathcal{D}_{t_{f}}^{q} \mathcal{P}
$$

with

$$
\left.\frac{\partial J}{\partial \mathcal{X}}\right|_{t=t_{f}}=0=\mathcal{P}\left(t_{f}\right) .
$$

Moreover, the optimality condition is given by the equation

$$
\left.\frac{\partial \mathcal{H}}{\partial \mathcal{U}}\right|_{\mathcal{U}^{*}}=0
$$

For the optimal control to lie within the admissible space $U_{a d}$, we project it onto the interval $[0.05,0.95]$. In the preceding theorem, we state the necessary optimality conditions.

Theorem 3. Given an optimal control $\mathcal{U}^{*}=$ $\left(u_{1}, u_{2}, u_{3}\right)$ and the state solution $\mathcal{X}^{*}=$ $(S, H, I, L, N)$ corresponding to 


$$
\left\{\begin{aligned}
{ }_{0}^{C} \mathcal{D}_{t}^{q} S(t) & =b^{q} N(t)-\beta^{q}\left(1-u_{1}(t)\right) \frac{S(t) I(t)}{N(t)} \\
& -\mu^{q} S(t), \\
{ }_{0}^{C} \mathcal{D}_{t}^{q} H(t) & =\beta^{q}\left(1-u_{1}(t)\right) \frac{S(t) I(t)}{N(t)} \\
& -\left(\alpha^{q}\left(1+u_{2}(t)\right)+\kappa^{q}+\mu^{q}\right) H(t) \\
& +p r^{q}\left(1-u_{3}(t)\right) I(t), \\
{ }_{0}^{C} \mathcal{D}_{t}^{q} I(t) & =\kappa^{q} H(t)-\left(r^{q}+\mu^{q}+d^{q}\right) I(t), \\
{ }_{0}^{C} \mathcal{D}_{t}^{q} L(t) & =\left(1-p\left(1-u_{3}(t)\right)\right) r^{q} I(t) \\
& +\alpha^{q}\left(1+u_{2}(t)\right) H(t)-\mu^{q} L(t), \\
{ }_{0}^{C} \mathcal{D}_{t}^{q} N(t) & =\left(b^{q}-\mu^{q}\right) N(t)-d^{q} I(t),
\end{aligned}\right.
$$

with $S(0)=S_{0}, H(0)=H_{0}, I(0)=I_{0}, L(0)=$ $L_{0}$ and $N(0)=N_{0}$ that minimize the objective functional (6), there exist adjoint variables $\mathcal{P}=\left(\lambda_{1}(t), \lambda_{2}(t), \lambda_{3}(t), \lambda_{4}(t), \lambda_{5}(t)\right)$ satisfying

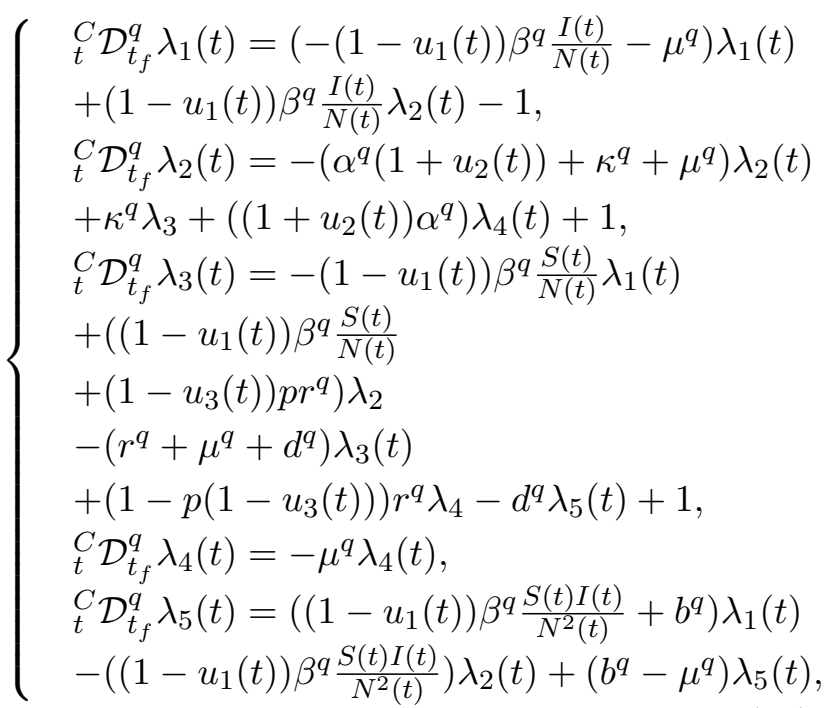

with transversality conditions

$$
\lambda_{i}\left(t_{f}\right)=0, \quad 1 \leq i \leq 5
$$

Moreover, the optimal control $\mathcal{U}^{*}=$ $\left(u_{1}(t), u_{2}(t), u_{3}(t)\right)$ is represented by

$$
\left\{\begin{array}{l}
u_{1}(t)=\min \left(\max \left(\left(\lambda_{2}(t)-\lambda_{1}(t)\right) \frac{\beta^{q} S(t) I(t)}{\omega_{1} N(t)}, 0.05\right), 0.95\right), \\
u_{2}(t)=\min \left(\max \left(\left(\lambda_{2}(t)-\lambda_{4}(t)\right) \frac{\alpha^{q} H(t)}{\omega_{2}}, 0.05\right), 0.95\right), \\
u_{3}(t)=\min \left(\max \left(\left(\lambda_{2}(t)-\lambda_{4}(t)\right) \frac{p r^{q} I(t)}{\omega_{3}}, 0.05\right), 0.95\right) .
\end{array}\right.
$$

\section{Numerical results}

In this section, we present some illustrative examples to observe the contribution of the fractional derivative to the choice of the control strategy. We apply 3 different control intervention approaches and their combinations: The first one (Case $C_{1}$ ) is distancing control $u_{1}(t)$, which is based on eliminating the contact between infectious and susceptible people. The second approach $\left(\right.$ Case $C_{2}$ ) is latent case finding control $u_{2}(t)$ which aims to treat high-risk latent class. The last strategy (Case $C_{3}$ ) is case holding control $u_{3}(t)$ which consists of some actions applied to eliminate the failure of the treatment.

We use the parameter values given in Table 1 which lead the reproduction number to be $\mathcal{R}_{0}=$ $\{2.4872,2.8507,3.2546\}$ for the fractional orders $q \in\{0.85,0.9,0.95\}$, respectively. Therefore, the infection will not disappear in the future if the initial conditions are taken close to the disease-free equilibrium point.

We fix the weight parameters as $\left\{\omega_{1}, \omega_{2}, \omega_{3}\right\}=$ $\left\{10^{6}, 10^{6}, 10^{5}\right\}$ following the work [30] and they denote the cost of implementing the corresponding control strategy. We solve the FOCP on the time interval [2015,2035] with a constant step size $\Delta t=0.004$. We discretize the FOCP using L1-method 34] and forward-backward sweep method is used as an optimization algorithm [35]. The initial subpopulations are taken as $S_{0}=$ 20027781, $H_{0}=9292101, I_{0}=621331$ and $L_{0}=$ 32006125 (Aurelio A. de los Reyes V, personal communication, June 26, 2018). We investigate the contribution of three intervention strategies and their combinations by measuring the reduction/increase in $I$ and $S$ compared to uncontrolled case in Table 3. All simulations are performed on a Windows 10 machine with Intel Core i7, 2.5 $\mathrm{GHz}$ and $16 \mathrm{~GB}$ using MATLAB R2016a. With the discretization mentioned above, the FOCP is solved for 430 seconds in case of triple controls, while the uncontrolled problem is solved in almost 25 seconds. Here, rather then the computational time, we will focus on the influence of the fractional order.

Before discussing an optimal control strategy, we obtain the numerical solution of TB model (7) without control, that is, $u_{1}=u_{2}=u_{3}=0$. We measure the values of $H(t)+I(t), I(t)$ and $S(t)$ in 2035 and we present these results in Table 2 . We observe an increasing risk of the disease due to a large number of infected people. 


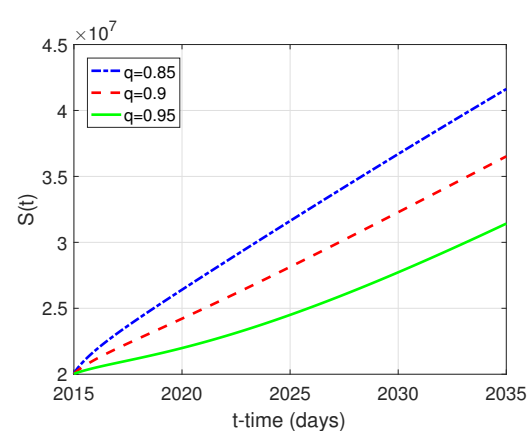

(a) Susceptible $S(t)$.

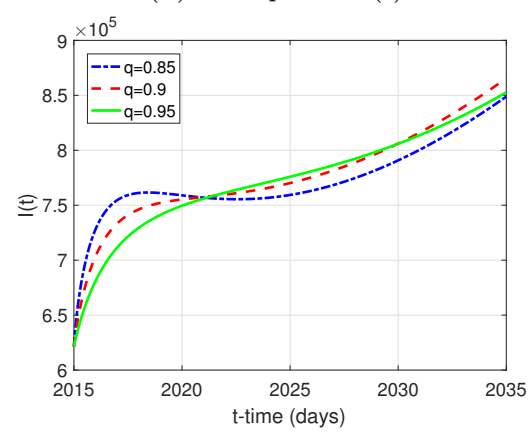

$(C)$ Infectious $I(t)$.

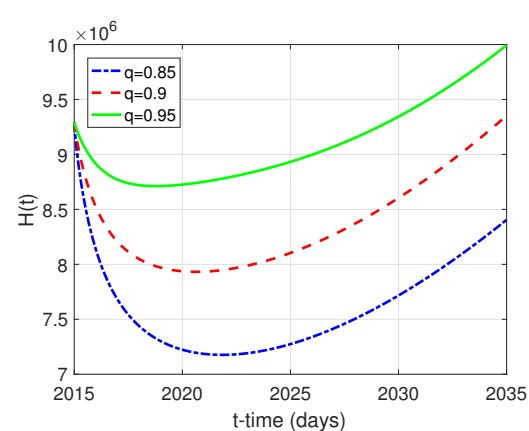

(b) High-risk latent $H(t)$.

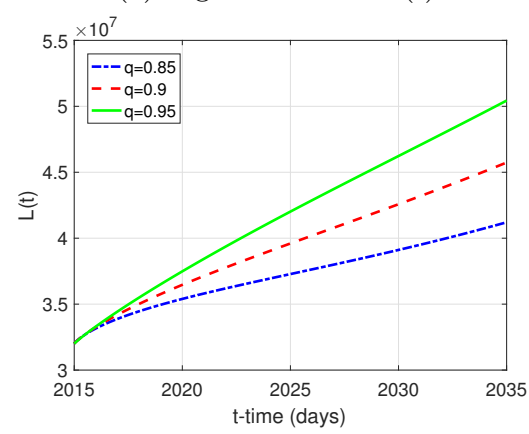

(D) Low-risk latent $L(t)$.

Figure 1. No control: Epidemiological classes.

Table 2. Uncontrolled case: Estimates for $H+I, I$ and $S$ in 2035 .

\begin{tabular}{llll}
\hline$\alpha$ & $H(2035)+I(2035)$ & $I(2035)$ & $S(2035)$ \\
\hline 0.85 & $9.2539 \mathrm{e}+06$ & $8.4889 \mathrm{e}+05$ & $4.1626 \mathrm{e}+07$ \\
0.9 & $1.0218 \mathrm{e}+07$ & $8.6547 \mathrm{e}+05$ & $3.6519 \mathrm{e}+07$ \\
0.95 & $1.0846 \mathrm{e}+07$ & $8.5268 \mathrm{e}+05$ & $3.1422 \mathrm{e}+07$ \\
\hline
\end{tabular}

Moreover, four epidemiological classes are shown in Fig. 1. We observe that infectious $I(t)$ and high-risk latent $H(t)$ populations increase over time which underlines the requirement of an efficient control strategy to eliminate the disease.

Therefore, we set the optimal control strategy to minimize the difference between uninfected and infected individuals with the following the cost functional:

$$
\begin{aligned}
& \min _{\left(u_{1}, u_{2}, u_{3}\right) \in U_{a d}} J\left(u_{1}, u_{2}, u_{3}\right)=\int_{0}^{t_{f}}(H(t)+I(t) \\
& \left.-S(t)+\frac{\omega_{1}}{2} u_{1}^{2}(t)+\frac{\omega_{2}}{2} u_{2}^{2}(t)+\frac{\omega_{3}}{2} u_{3}^{2}(t)\right) d t
\end{aligned}
$$

We immediately observe that cases $C_{1}, C_{12}$ and $C_{123}$ lead to the highest reduction in $I$ and increase in $S$. In other words, distancing control is the most efficient choice to reduce the number of infected people which leads to an increase in the susceptible individuals. Additionally, the case $C_{3}$, namely case holding control, is the least efficient choice. It means that some efforts to eliminate the failure of the treatment cannot be successful without any supportive strategy. Moreover, case $C_{2}$ denoting latent case finding control is the second most effective approach. However, its contribution can be boosted with distancing control. Since the contribution of case holding control is limited, there is not a big difference between $C_{12}$ and $C_{123}$ in the reduction of $I$, while increase in $S$ is almost the same for the cases $C_{1}, C_{12}$ and $C_{123}$. On the other hand, as we increase the order of the fractional derivative $q$, we observe a positive change in both reduction in $I$ and increase in $S$. It can be thought as the contribution of the memory effect. If we add more information about the history to the model, which corresponds to a higher value of $q$, then the success of the treatment will be more visible.

As some illustrative results, we depict the epidemiological classes for Case $C_{123}$, namely the combination of three control strategies, in Fig. 2 , We observe that the number of susceptible individuals is higher than one for the uncontrolled case, while there is a decline in the number of infected people. It means that the control strategy works well and the figures are compatible with the aim behind the FOCP.

In addition, we present the optimal controls $u_{1}, u_{2}$ and $u_{3}$ in Fig. 3. We see that the control lies between the predefined box constraints. As time passes, a smaller control (compared to initial time) is needed. Among these three different control strategies, distancing control is the most effective one to eliminate the disease. 


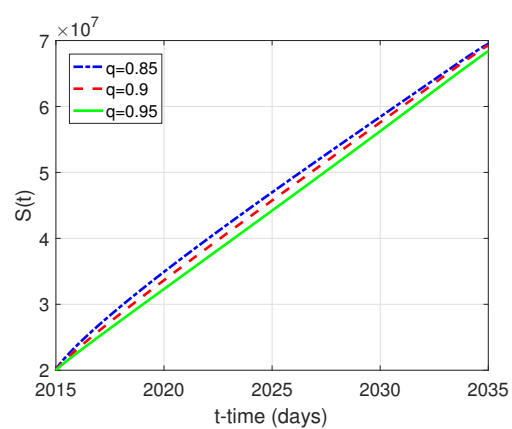

(a) Susceptible $S(t)$.

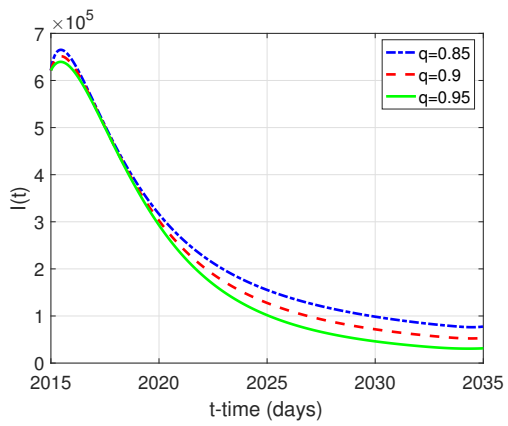

(c) Infectious $I(t)$.

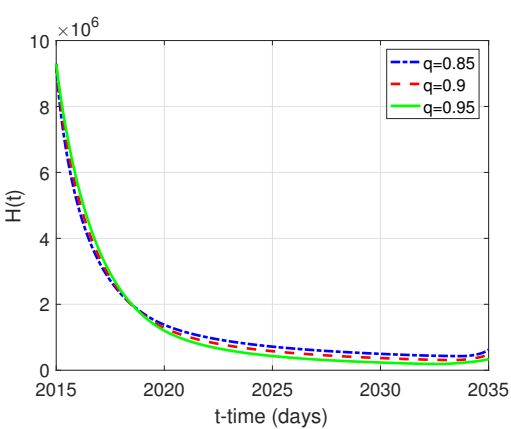

(b) High-risk latent $H(t)$.

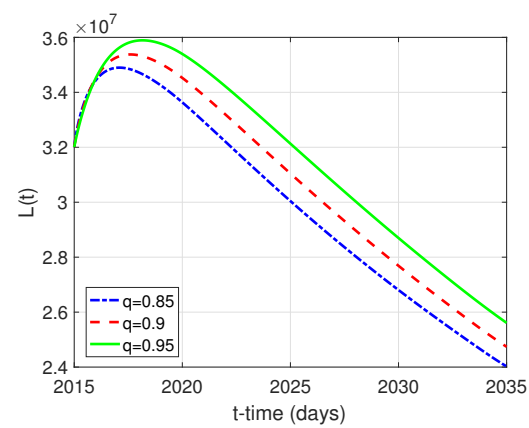

(d) Low-risk latent $L(t)$.

Figure 2. Case $C_{123}$ : Epidemiological classes.

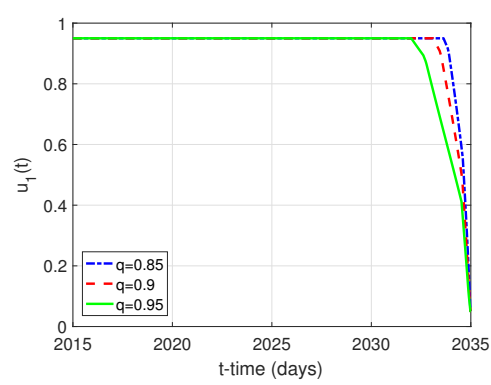

(a) Control $u_{1}(t)$.

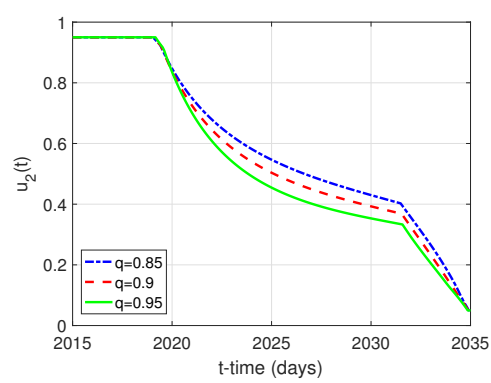

(b) Control $u_{2}(t)$.

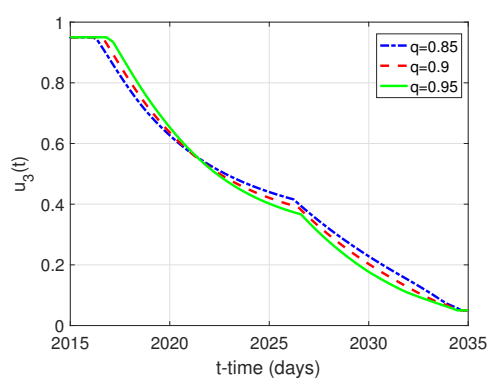

(c) Control $u_{3}(t)$.

Figure 3. Case $C_{123}$ : Optimal controls $u_{1}(t), u_{2}(t)$ and $u_{3}(t)$.

\section{Summary and conclusion}

In this study, we investigate an OCP governed by a TB model with Caputo time fractional derivative. We justify that the solution is nonnegativity and bounded from above and the optimality system is derived based on the Hamiltonian. We compare three different control strategies and their combinations, namely, distancing control, latent case finding and case holding control. We presented some numerical results to underline the contribution of the fractional order and the choice of the intervention strategy. We observe that the cases $C_{1}, C_{12}$ and $C_{123}$ lead to the most reduction in the number of infected people and increase in the susceptible individuals. Moreover, as we increase the order of the fractional derivative, optimal control strategies become more effective.

\section{Acknowledgments}

The author thanks the anonymous referees for their helpful suggestions.

\section{References}

[1] World Health Organization. (2017). Tuberculosis fact sheet. Geneva, Switzerland. Available from https://www.who.int/tb/publications /global_report/gtbr2017_main_text.pdf. [Accessed 6 July 2018].

[2] Feng, Z., Castillo-Chavez, C., \& Capurro, A.F. (2000). A model for tuberculosis with exogenous reinfection. Theoretical Population Biology, 57 (3), 235-247.

[3] Small, P.M., \& Fujiwara, P.I. (2001). Management of tuberculosis in the United States, 
Table 3. Estimates for $I(t)$ and $S(t)$ in 2035 and the corresponding reduction/increase.

\begin{tabular}{|c|c|c|c|c|}
\hline $\begin{array}{l}\text { Case } C_{1} \\
\alpha\end{array}$ & $I(2035)$ & (Reduction \%) & $S(2035)$ & (Increase \%) \\
\hline 0.85 & $1.0862 \mathrm{e}+05$ & $\% 87.20$ & $6.9310 \mathrm{e}+07$ & $\% 66.51$ \\
\hline 0.9 & $7.9264 \mathrm{e}+04$ & $\% 90.84$ & $6.9068 \mathrm{e}+07$ & $\% 89.13$ \\
\hline 0.95 & $5.2400 \mathrm{e}+04$ & $\% 93.85$ & $6.8138 \mathrm{e}+07$ & $\% 116.85$ \\
\hline \multicolumn{5}{|l|}{ Case $C_{2}$} \\
\hline$\alpha$ & $I(2035)$ & (Reduction \%) & $S(2035)$ & (Increase \%) \\
\hline 0.85 & $2.9670 \mathrm{e}+05$ & $\% 65.05$ & $5.4971 \mathrm{e}+07$ & $\% 32.06$ \\
\hline 0.9 & $2.8429 \mathrm{e}+05$ & $\% 67.15$ & $5.1607 \mathrm{e}+07$ & $\% 41.32$ \\
\hline 0.95 & $2.6649 \mathrm{e}+05$ & $\% 68.75$ & $4.7425 \mathrm{e}+07$ & $\% 50.93$ \\
\hline \multicolumn{5}{|l|}{ Case $C_{3}$} \\
\hline 0.85 & $8.3356 \mathrm{e}+05$ & $\% 1.81$ & $4.2935 \mathrm{e}+07$ & $\% 3.14$ \\
\hline 0.9 & $8.4981 \mathrm{e}+05$ & $\% 1.81$ & $3.7681 \mathrm{e}+07$ & $\% 3.18$ \\
\hline 0.95 & $8.3386 \mathrm{e}+05$ & $\% 2.21$ & $3.2304 \mathrm{e}+07$ & $\% 2.81$ \\
\hline \multicolumn{5}{|l|}{ Case $C_{12}$} \\
\hline$\alpha$ & $I(2035)$ & (Reduction \%) & $S(2035)$ & (Increase \%) \\
\hline 0.85 & $7.8062 \mathrm{e}+04$ & $\% 90.80$ & $6.9499 \mathrm{e}+07$ & $\% 66.96$ \\
\hline 0.9 & $5.3686 \mathrm{e}+04$ & $\% 93.80$ & $6.9293 \mathrm{e}+07$ & $\% 89.75$ \\
\hline 0.95 & $3.1645 \mathrm{e}+04$ & $\% 96.29$ & $6.8405 \mathrm{e}+07$ & $\% 117.70$ \\
\hline \multicolumn{5}{|l|}{ Case $C_{13}$} \\
\hline$\alpha$ & $I(2035)$ & (Reduction \%) & $S(2035)$ & (Increase \%) \\
\hline 0.85 & $1.0653 \mathrm{e}+05$ & $\% 87.45$ & $6.9317 \mathrm{e}+07$ & $\% 66.52$ \\
\hline 0.9 & $7.7363 \mathrm{e}+04$ & $\% 91.06$ & $6.9080 \mathrm{e}+07$ & $\% 89.16$ \\
\hline 0.95 & $5.0710 \mathrm{e}+04$ & $\% 94.05$ & $6.8151 \mathrm{e}+07$ & $\% 116.89$ \\
\hline \multicolumn{5}{|l|}{ Case $C_{23}$} \\
\hline$\alpha$ & $I(2035)$ & (Reduction \%) & $S(2035)$ & (Increase \%) \\
\hline 0.85 & $2.9065 \mathrm{e}+05$ & $\% 65.76$ & $5.5203 \mathrm{e}+07$ & $\% 32.62$ \\
\hline 0.9 & $2.7810 \mathrm{e}+05$ & $\% 67.87$ & $5.1875 \mathrm{e}+07$ & $\% 42.05$ \\
\hline 0.95 & $2.6041 \mathrm{e}+05$ & $\% 69.46$ & $4.7717 \mathrm{e}+07$ & $\% 51.86$ \\
\hline \multicolumn{5}{|l|}{$\overline{\text { Case } C_{123}}$} \\
\hline 0.85 & $7.7656 \mathrm{e}+04$ & $\% 90.85$ & $6.9502 \mathrm{e}+07$ & $\% 66.97$ \\
\hline 0.9 & $5.3334 \mathrm{e}+04$ & $\% 93.84$ & $6.9297 \mathrm{e}+07$ & $\% 89.76$ \\
\hline 0.95 & $3.1380 \mathrm{e}+04$ & $\% 96.32$ & $6.8410 \mathrm{e}+07$ & $\% 117.71$ \\
\hline
\end{tabular}

New England Journal of Medicine, 345 (3), 189-200.

[4] Bailey, N.T., et al., (1975). The mathematical theory of infectious diseases and its applications. Charles Griffin \& Company Ltd, 5a Crendon Street, High Wycombe, Bucks HP13 6LE.

[5] Waaler, H., Geser, A., \& Andersen, S. (1962). The use of mathematical models in the study of the epidemiology of tuberculosis. American Journal of Public Health and the Nations Health, 52 (6), 1002-1013.

[6] Hethcote, H.W. (2000). The mathematics of infectious diseases. SIAM Review, 42 (4), 599653.

[7] Choisy, M., Guégan, J.F., \& Rohani, P. (2007). Mathematical modeling of infectious diseases dynamics. Encyclopedia of infectious diseases: Modern methodologies, 379-404.

[8] Castillo-Chavez, C., \& Feng, Z. (1997) To treat or not to treat: the case of tuberculosis. Journal of Mathematical Biology, 35 (6), 629-656.

[9] Castillo-Chávez, C., Feng, Z., et al. (1998). Mathematical models for the disease dynamics of tuberculosis. in: G. S. Mary Ann Horn, G. Webb (Eds.), Advances in Mathematical Population Dynamics-Molecules, Cells and Man, Vanderbilt University Press, 117-128.

[10] Liu, L., Zhao, X.Q., \& Zhou, Y., (2010). A tuberculosis model with seasonality. Bulletin of Mathematical Biology, 72 (4), 931-952. 
[11] Podlubny, I. (1999). Fractional differential equations, Vol. 198 of Mathematics in Science and Engineering, Academic Press, Inc., San Diego, CA, An introduction to fractional derivatives, fractional differential equations, to methods of their solution and some of their applications.

[12] Yang, X.J., \& Machado, J.A.T. (2017). A new fractional operator of variable order: application in the description of anomalous diffusion. Physica A: Statistical Mechanics and its Applications, 481, 276-283.

[13] Moghaddam, B.P., \& Machado, J.A.T. (2017). Extended algorithms for approximating variable order fractional derivatives with applications. Journal of Scientific Computing, 71 (3), 1351-1374.

[14] Moghaddam,B.P., \& Machado, J.A.T. (2017). SM-algorithms for approximating the variable-order fractional derivative of high order. Fundamenta Informaticae, 151 (1-4), 293-311.

[15] Koca, I. (2018). Analysis of rubella disease model with non-local and non-singular fractional derivatives. An International Journal of Optimization and Control: Theories and Applications, 8 (1), 17-25.

[16] Sweilam, N.H., Al-Mekhlafi, S.M., \& Assiri, T.A.R. (2017). Numerical study for time delay multistrain tuberculosis model of fractional order. Complexity, 2017, Article ID 1047381, 14 pages.

[17] Wojtak, W., Silva, C.J., \& Torres, D.F. (2018). Uniform asymptotic stability of a fractional tuberculosis model. Mathematical Modelling of Natural Phenomena, 13 (1), 9.

[18] Pinto, C.M.A., \& Carvalho, A.R.M. (2017). The HIV/TB coinfection severity in the presence of TB multi-drug resistant strains. Ecological complexity, 32, 1-20.

[19] Carvalho, A.R.M., \& Pinto, C.M.A. (2018). Non-integer order analysis of the impact of diabetes and resistant strains in a model for TB infection. Communications in Nonlinear Science and Numerical Simulation, 61, 104-126.

[20] De Pillis, L.G., \& Radunskaya, A. (2001). A mathematical tumor model with immune resistance and drug therapy: An optimal control approach. Computational and Mathematical Methods in Medicine, 3 (2), 79-100.

[21] Okosun, K.O., Ouifki, R., \& Marcus, N. (2011). Optimal control analysis of a malaria disease transmission model that includes treatment and vaccination with waning immunity. Biosystems, 106 (2-3), 136-145.
[22] Area, I, Ndä̈rou, F., Nieto, J.J., Silva, C.J., \& Torres, D.F.M. (2018). Ebola model and optimal control with vaccination constraints. Journal of Industrial \&3 Management Optimization, 14 (2), 427-446.

[23] Hamdache, A., Saadi, S., \& Elmouki, I. (2016). Free terminal time optimal control problem for the treatment of HIV infection. An International Journal of Optimization and Control: Theories \& Applications, 6 (1), 3351.

[24] Sweilam, N.H., \& Al-Mekhlafi, S.M. (2016). On the optimal control for fractional multistrain TB model. Optimal Control Applications and Methods, 37 (6), 1355-1374.

[25] Silva, C.J., \& Torres, D.F.M. (2013). Optimal control for a tuberculosis model with reinfection and post-exposure interventions. Mathematical Biosciences, 244 (2), (2013) 154-164.

[26] Bowong, S. (2010). Optimal control of the transmission dynamics of tuberculosis. Nonlinear Dynamics, 61 (4), 729-748.

[27] Moualeu, D.P., Weiser, M., Ehrig, R., \& Deuflhard, P. (2015). Optimal control for a tuberculosis model with undetected cases in Cameroon. Communications in Nonlinear Science and Numerical Simulation, 20 (3), 986-1003.

[28] Jung, E., Lenhart, S., \& Feng, Z. (2002). Optimal control of treatments in a two-strain tuberculosis model. Discrete and Continuous Dynamical Systems Series B, 2 (4), 473-482.

[29] Silva, C.J., \& Torres, D.F.M. (2015). Optimal control of tuberculosis: A review, in: $D y$ namics, Games and Science, Springer, 701722 .

[30] Kim, S., Aurelio, A. de Los Reyes V., \& Jung, E. (2018). Mathematical model and intervention strategies for mitigating tuberculosis in the Philippines. Journal of Theoretical Biology, 443, 100-112.

[31] Odibat, Z.M., \& Shawagfeh, N.T. (2007). Generalized Taylor's formula. Applied Mathematics and Computation, 186 (1), 286-293.

[32] Diethelm, K. (2013). A fractional calculus based model for the simulation of an outbreak of dengue fever. Nonlinear Dynamics, 71 (4), 613-619.

[33] Pontryagin, L., Boltyanskii, V., Gamkrelidze, R., \& Mishchenko, E. (1962). The Mathematical Theory of Optimal Processes. John Wiley \& Sons, New York. 
[34] Lin, Y., \& Xu, C. (2007). Finite difference/spectral approximations for the timefractional diffusion equation. Journal of Computational Physics, 225 (2), 1533-1552.

[35] Lenhart, S., Workman, J.T. (2007). Optimal Control Applied to Biological Models. Chapman \& Hall, CRC Press.
Tuğba Akman Yıldız achieved her PhD degree in Scientific Computing Program at Institute of Applied Mathematics, Middle East Technical University in 2015. She has been working as an assistant professor at UTAA since 2016. Her research interests are numerical solutions of differantial equations, optimal control problems and model order reduction methods.

An International Journal of Optimization and Control: Theories \& Applications (http://ijocta.balikesir.edu.tr)

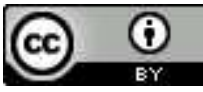

This work is licensed under a Creative Commons Attribution 4.0 International License. The authors retain ownership of the copyright for their article, but they allow anyone to download, reuse, reprint, modify, distribute, and/or copy articles in IJOCTA, so long as the original authors and source are credited. To see the complete license contents, please visit http://creativecommons.org/licenses/by/4.0/. 